\title{
Factores de riesgo suicida en población adolescente
}

\section{Suicide risk factors in adolescent population}

\author{
PACHECO-AMIGO, Beatriz Mabel†**\& TRONCOSO-PACHECO, Rómulo Enrique \\ Universidad Autónoma de Zacatecas, México \\ Universidad Ciudadana de Nuevo León, México \\ ID $1^{\text {er }}$ Autor: Beatriz Mabel, Pacheco-Amigo / ORC ID: 0000-0002-8053-3506, Researcher ID Thomson: P-7758-2016, \\ CVU CONACYT ID: 5147251
}

ID $1^{\text {er }}$ Coautor: Rómulo Enrique, Troncoso-Pacheco / ORC ID: 0000-0002-2074-9743, CVU CONACYT ID: 1096495

\section{Resumen}

Objetivo: El objetivo de la investigación es analizar cartas póstumas que permiten describir pensamientos orientados al déficit de adaptabilidad del menor, identificando los factores de riesgo más usuales presentados en los adolescentes. Metodología: El paradigma utilizado es mediante un enfoque cualicuantitativo - enfoque mixto y diseño no experimental, mediante un estudio transversal que permitió establecer la prevalencia de frecuencias de los indicadores de riesgo. La muestra fueron cartas póstumas de cinco adolescente que acentaron el acto suicida con un promedio de edad de 14 años con 8 meses en el año 2020. Por esta razón la muestra fue con características de intencionalidad, realizando una integración de categorías de las cartas póstumas permitiendo establecer concepciones teóricas e implicaciones prácticas, evaluándolas por análisis contextual mediante fase de programación de Python y librerías de Matplotlib. Contribución: Una de las principales contribuciones de la investigación es abordar los factores de riesgo del adolescente desde cartas póstumas con la finalidad de establecer lineamientos de intervención de salud mental para la prevención de acto suicida en menores.

Suicidio, Factores de riesgo, Adolescentes, Cartas póstumas

\begin{abstract}
Objectives: The objective of the research is to analyze posthumous letters that allow describing thoughts oriented to the child's adaptability deficit, identifying the most common risk factors presented in adolescents. Methodology: The paradigm used is through a qualiquantitative approach - mixed approach and nonexperimental design, through a cross-sectional study that allowed establishing the prevalence of frequencies of risk indicators. The sample consisted of posthumous letters from five adolescents who accentuated the suicidal act with an average age of 14 years and 8 months, in the year 2020. For this reason, the sample was of intentionality characteristics, integrating the categories of the posthumous letters allowing establish theoretical conceptions and practical implications, evaluating them by contextual analysis through Python programming phase and Matplotlib libraries. Contribution: One of the main contributions of the research is to address the risk factors of adolescents from posthumous letters in order to establish mental health intervention guidelines for the prevention of suicidal acts in minors.
\end{abstract}

Suicide, Risk factors, Adolescent, Posthumous letters

Citación: PACHECO-AMIGO, Beatriz Mabel \& TRONCOSO-PACHECO, Rómulo Enrique. Factores de riesgo suicida en población adolescente. Revista de Filosofía y Cotidianidad. 2020. 6-19: 1-6

\footnotetext{
* Correspondencia del Autor (bpachecoamigo@yahoo.com.mx)

$\dagger$ Investigador contribuyendo como primer Autor.
} 


\section{Introduction}

El suicidio en los adolescentes se empieza a establecer como factor de riesgo en diversas áreas de desarrollo, incluyendo el impacto en salud pública a nivel mundial, dato según la Organización Mundial de la Salud (2019), citando cifras, es la tercer causa de muerte entre edades de 15 a 19 años, asimismo, es un problema de salud global que se presenta en los jóvenes. Razón por la que es imperante la necesidad de abordar la problemática de salud mental en dicha población.

Por otra parte, en la República Mexicana en el 2020, la depresión es la primer causa de conducta suicida como a su vez, el suicidio es la tercer causa de muerte en niños y adolescentes (Instituto Nacional de Estadística y Geografía e informática- INEGI; 2020).

La importancia de la salud mental en los adolescentes es por la presencia de trastornos mentales estimado a nivel mundial que alcanzan el $20 \%$ de la población sin ser diagnosticada como tampoco abordado en tratamiento, cuya morbilidad se establece en edades antes de los 14 años (Organización Mundial de la Salud 2020).

El comportamiento suicida como acto de voluntad personal e individual, presenta diversas interpretaciones sobre el acto mismo, una de las principales son las psicológicas que al estar en un estado de constante cambio pueden desarrollar patrones de conductas desadaptadas que aumenta la posibilidad de conductas de autodestrucción. En otras palabras, el acto suicida se establecen condiciones específicas que permiten aumentar conductas de riesgo y, por otra parte, se aunan aquellas que pueden ser detonadores de conducta destructiva ( Quintanar, 2007).

Desde la perspectiva clínica, hace poco se integra el diagnóstico de Trastorno de desregulación disruptiva del estado de ánimo y en sus siglas TDDEA; cuadro clínico que afecta a niños y adolescentes, permitiendo identificar problemas conductuales y emocionales serios que se caracteriza por una irritabilidad de tipo no episódica acompañada por un descontrol conductual (Manual diagnóstico y Estadístico de los Trastornos Mentales, 2018).
Sin embargo, la prevalencia de los trastornos depresivos tiene un impacto importante a nivel de género estimando que es de 4 a 8 en mujeres posteriores a la pubertad, como también, en varones presentan un aumento de 6 a 8 en síntomas de estados distímicos, secundando en los jóvenes el surgimiento de síntomas subsindrómicos que afecta el deterioro de sus áreas de funcionamiento como también el riesgo de padecer sintomatología relacionadas a psicopatologías específicas a futuro (Royo y Martínez, 2012).

Por otra parte, el Hospital Psiquiátrico Infantil de México, hace referencia que existe una estimación de frecuencia del 3.3 en adolescentes que mantienen una edad de 13 a 18 años en proporción a 100 mil habitantes, a diferencia de los niños suicidas son de 0.3 sobre cada 100 mil habitantes (Chávez, s/f).

Con los datos expuestos se puede analizar los efectos asociados a cuadros clínicos específicos orientados a la conducta suicida, por esta razón, la preocupación de realizar estudios en adolescentes para identificar factores de riesgo en los menores y brindar posibles alternativas de intervención basado en un previo diagnóstico de análisis contextual en cartas póstumas. De esta forma, el valor que se agrega a la investigación es la realización de un análisis contextual mediante un lenguaje de programación de Python y de librerías Matplotlib, que auxilió a integrar la organización estadística de categorías con sus respectivos indicadores en cartas póstumas de adolescentes que incurrieron en el acto suicida, para poder brindar alternativas de intervención y sobre todo de prevención del acto estudiado.

\section{Referentes teóricos de la adolescencia}

La adolescencia como proceso de formación y maduración de la personalidad, no sólo aborda estándares generales sociales sino que también se establecen mecanismos psicológicos específicos del menor, de esta forma, como lo refiere Aberastury (1971) la adolescencia es un proceso de corte evolutivo universal, en éste se presentan una serie de desprendimiento que podrán favorecer o dificultar dichos procesos en el menor, mismos que auxilian a la preparación para alcanzar la madurez de un adulto-morfismo. 
Sin embargo, para llegar a estos estadíos es indispensable la superación de diversos retos que conlleva a logros en el desarrollo, y sin lugar a duda, existirán conductas de asimilación de conocimientos como también conductas inapropiadas sin llegar a realizar conductas de autolesión. Uno de los principales problemas del adolescente son las conductas de regulación, en ellas, se integran un conjunto de características psicológicas que se producen en el marco del desarrollo que son el duelo por su cuerpo, el duelo por el rol e identidad y el duelo de la infancia (Aberástury et col, 1971). El obstáculo radica, el cómo se establece esta regulación de duelos que no permite abordar las siguientes fases de desarrollo evolutivo.

La conceptualización adecuada del suicidio no sólo aborda estructuras clínicas sino también se deben estudiar desde una perspectiva socio-cultural; por ello, el acto suicida se puede considerar como uno de los mayores fracasos de adaptación que puede manifestar el adolescente; a causa de que, todo acto con estas características están desarrolladas y ejecutadas en un acto de crisis, referido por Quintanar (2007), mismas que se pueden representar en tipos de conducta suicida que son:

\begin{tabular}{|l|l|}
\hline \multicolumn{1}{|c|}{$\begin{array}{c}\text { Conducta } \\
\text { Suicida }\end{array}$} \\
\hline Gesto suicida & $\begin{array}{l}\text { Al ingerir sustancias o realizar } \\
\text { lesiones que no ocasionan la muerte } \\
\text { en el sujeto. }\end{array}$ \\
\hline $\begin{array}{l}\text { Intento } \\
\text { suicida }\end{array}$ & $\begin{array}{l}\text { Acto cuyo objetivo es la mortandad, } \\
\text { pero es ineficiente al objetivo. }\end{array}$ \\
\hline $\begin{array}{l}\text { Suicidio } \\
\text { consumado }\end{array}$ & $\begin{array}{l}\text { Se logra el objetivo de la acción } \\
\text { voluntaria o por no recibir atención en } \\
\text { tiempo. }\end{array}$ \\
\hline
\end{tabular}

Table 1 Tipos de conducta suicida

Fuente: Elaboración Propia, extraído de Quintanar (2007)

\section{Factores de riesgo para la conducta suicida}

Dentro de un marco estricto de conceptualización psicodinámica el acto suicida, el sujeto se encuentra en un dolor que no puede controlar y para finalizarlo se llega a ejercer el acto; por esa condición, el suicida (independientemente de la edad), no es aquel que no quiere vivir sino que le faltan alternativas para poder vivir (Pacheco, 2019); por ello, siempre es un acto con una formación lógica y estructurada de la realidad, que se ven afectadas por factores de riesgos (Quintanar, 2007)
Una de las primeras causas como factor de riesgo es la presencia de cuadros depresivos, que, en el caso de los adolescentes se asocian con alteraciones en las áreas cognitivas disminuyendo la integración en el área académica, fallas de memoria, disminuyendo de manera significativa la capacidad de atención y concentración (Royo et col, 2012). En éste, se pueden presentar síntomas atípicos al cuadro clínico del adulto refiriendo hipersomnia, aumento de apetito, indiferencia, aplanamiento emocional, letargia entre otros, que potencia la aparición de otros cuadros con mayor significancia y alteración en las Actividades de la Vida Diaria (Pacheco, 2020).

Se aúna, otro factor de riesgo que es el uso y abuso de sustancias tóxicas (uso de alcohol o de algún uso de droga aún cuando sea con fines recreativos), que a largo plazo son potenciadores de síntomas y precursores de síntomas subsecuentes de estados patognomónicos. Así, al aglutinar problemas en el contexto familiar, problemas de relaciones afectivas especialemente amorosas o padecimiento de enfermedad INEGI (2020), son factores activadores para poder realizar el acto suicida. A su vez, en el adolescente se requieren otras necesidades afectivas, mismas que se pueden orientar a factores de riesgos como factores precipitantes para el suicidio destacando el disgusto familiar, enfermedades mentales o físicas, diversos tipos de maltrato infantil, reprobación de exámenes, mala condición social con los pares y con porcentualidad baja alguna causa amorosa (INEGI, 2020).

\section{Cartas póstumas}

La importancia de valorar las cartas suicidas o cartas póstumas, son porque permiten valorar la gravedad y efectos de malestar psíquicos del menor, a su vez, establece conflictos y dinámicas que se articulan en su decisión para llevar a cabo el acto. De esta manera los mensajes póstumos se consideran un análisis del discurso, que permite identificar problemas de corte social y que, mediante el análisis, permitan auxiliar en propuestas que impacten de manera favorable los grupos con mayor vulnerabilidad de los adolescentes; si bien es cierto, son pocas las ocasiones que presentan cartas de estas condiciones, teniendo un estimativo que sólo el $10 \%$ de los jóvenes dejan este tipo de construcción linguística (Chávez, s/f). 
Asimismo, la elaboración de estas cartas $\mathrm{y}$ en su discurso se realiza una forma de comunicación, anunciado como " la lengua se efectúa en una instancia de discurso, que emana de un locutor" , citado por Aguilar y Neri (2018).

De esta manera, (siguiendo con los mismos autores), el suicida en sus cartas póstumas no existe un diálogo, por lo que se analiza en esta investigación una serie de constructos que originaron categorías en un marco contextual de cartas póstumas.

Por otra parte, Romero (2015), hace referencia que las cartas o notas póstumas , expresan un sentimiento de pobreza afectiva ante la vida, añadiendo un déficit de planes a futuro sobre la vida del adolescente, señalando, en términos clínico, los jóvenes se encuentran con frecuencia en estados alterados del ánimo que como sintomatología aparece la desesperanza ligado a episodios depresivos, aunado a un manejo inadecuado de la agresión, lo que no permite realizar una adecuada gestión de sus afectos y sentimientos por el nivel de desarrollo en el que se encuentran, razón por la cual se le dificulta identificar alternativas apropiadas para la resolución de problemas (Pacheco, 2019).

\section{Metodología}

La metodología utilizada fue bajo un paradigma cuali-cuantitativo; en el primero se utilizó un análisis contextual de las cartas póstumas que permitió establecer la construcción de indicadores para poder evaluar mediante unidad de análisis única (cartas póstumas) de adolescentes entre 10 y 17 años, cuyo promedio de edad fue de 14 años con 8 meses estableciendo categorías de la cualidad del objeto de estudio; el método de utilización del marco evaluativo fue el análisis contextual, lo que orientó a una muestra de tipo no probabiblística de tipo intencionado.

Por esta razón el estudio en relación a su diseño es no experimental, de tipo analíticadescriptiva, utilizando exclusivamente fuentes de orden literario en cartas póstumas para poder realizar el análisis simbólico y el análisis de contenido como técnica propia de la investigación, para orientarlo al método contextual para originar datos cuantificables posteriormente.
Por este motivo el objetivo de la investigación es analizar cartas póstumas que permiten describir pensamientos orientados al déficit de adaptabilidad del menor, identificando los factores de riesgo más usuales presentados en los adolescentes.

Para soportar la fase cuantitativa de la investigación, mediante la integración de categorías del análisis contextual, se auxilió de un lenguaje de programación Python y librerías de Matplotlib, que permitió plotear la información organizada en fase cuantitativa para el desarrollo de frecuencias de palabras claves existentes en cartas póstumas, desde una postura previamente cualitativa.

Posteriormente, se realizó un filtrado de puntuación en el texto con la finalidad de no arrojar texto basura; para proseguir con la búsqueda de palabras claves por caso analizado, almacenando la frecuencia de éstas en una matriz de 10 x 5, conformándose de la siguiente manera:

\begin{tabular}{|l|l|}
\hline \multicolumn{2}{|c|}{ Columna } \\
\hline Familia & $\begin{array}{l}\text { Conceptos en frecuencias } \\
\text { padrastro papá, hermanos, }\end{array}$ \\
\hline Cognición & $\begin{array}{l}\text { Valoración, fracaso, cabeza } \\
\text { hueca, estorbo. }\end{array}$ \\
\hline $\begin{array}{l}\text { Medio } \\
\text { Socio/Familiar }\end{array}$ & $\begin{array}{l}\text { Entendimiento, comprensión, } \\
\text { gente, gente como yo }\end{array}$ \\
\hline Conceptos evolutivos & Madurar y nacer \\
\hline Sentimiento/emoción & $\begin{array}{l}\text { Perdón, querer, sufrir, soledad, } \\
\text { agradecimiento, gracias, } \\
\text { fracaso }\end{array}$ \\
\hline
\end{tabular}

Tabla 2 Integración de Columnas por categorías Fuente: Elaboración Propia

Posteriormente se sumaron todos los valores por columna en un tamaño de vector 5 , con la finalidad de originar el resultado de frecuencias globales por categoría. Posteriormente se utilizó la librería de Matplotlib para poder graficar cada una de las categorías en base a los indicadores, originando una gráfica de pastel.

\begin{tabular}{|l|r|}
\hline Sujetos & Frecuencias de indicadores \\
\hline 1 & $4,0,0,1,8$ \\
\hline 2 & $1,0,0,0,2$ \\
\hline 3 & $2,0,1,0,2$ \\
\hline 4 & $3,0,1,0,4$ \\
\hline 5 & $2,1,1,0,0$ \\
\hline
\end{tabular}

Tabla 3 Número de sujetos y frecuencia de indicadores Fuente: Elaboración Propia 


\section{Results}

$\mathrm{Al}$ analizar las cartas póstumas se buscaron las categorías establecidas de las cuales se detectaron e identificaron los indicadores como palabras claves que correspondieran en el contexto del acto suicida y cuyas frecuencias fueron:

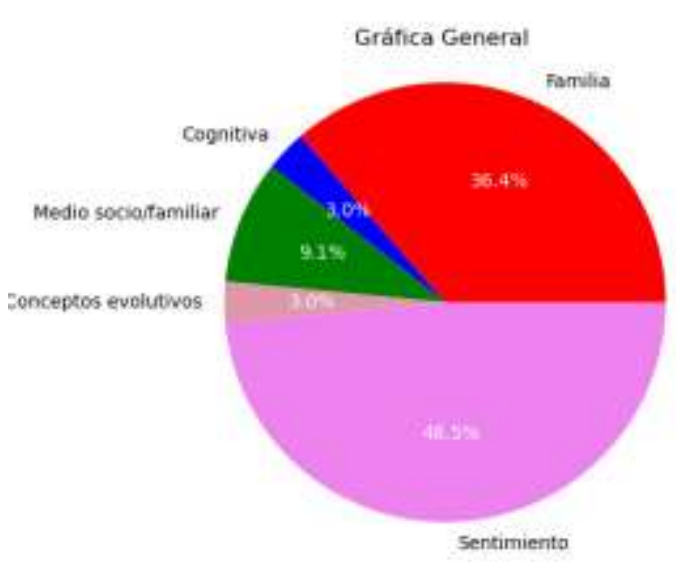

Gráfica 1 Frecuencia general de categorías identificadas

Nota: En términos de porcentualidad las tres mayores categorías de análisis de riesgo fueron lo relacionado a lo sentimientos, relaciones con familiar inmediato y rubro socio/familiar relacionado al vínculo (ver tabla 2)

\begin{tabular}{|l|r|}
\hline \multicolumn{1}{|c|}{ Categorías } & \multicolumn{1}{c|}{$\begin{array}{c}\text { Frecuencias de } \\
\text { categorías (\%) }\end{array}$} \\
\hline Sentimientos & $48.5 \%$ \\
\hline Familia & $36.4 \%$ \\
\hline Medio socio/familiar & $9.1 \%$ \\
\hline Cognitiva & $3 \%$ \\
\hline Concepto evolutivo & $3 \%$ \\
\hline
\end{tabular}

Tabla 4 Generalidad de frecuencia

\section{Thanks}

Se hace un agradecimiento a las personas que brindaron las cartas de uso público para poder realizar el análisis.

\section{Conclusions}

1. Se presentan con mayor frecuencia sentimientos de sufrimiento, perdón, fracaso ante los ideales de los padres, sin embargo, se presentan sentimientos de ambivalencia como lo son el agradecimiento ante la posibilidad de vida. Con ésto, se demuestra a nivel teórico la ambivalencia que se presenta en los menores, como también niveles de desesperanza que no permite establecer vías alternas de solución de problema.
Seguido a la mayor frecuencia se integran todas las relaciones de corte exclusivo familiar, situación que hace referencia a la queja sobre ellos, pero la gran mayoría (casi en su totalidad), dentro del análisis realiza la petición de perdón hacia sus padres, específicamente a la madre. Por otra parte, solamente se detectó un hallazgo, donde implícitamente era una queja hacia la madre pero relacionado a la vinculación con el padrastro y no se encontraron datos de suicidio por formaciones de vínculos amorosos en las cartas analizadas.

2. La frecuencia del medio socio/familiar se integraron aquellos indicadores de palabras claves, en los que se sentían incomprendidos dentro del hogar como fuera de él, alcanzando un $9.1 \%$, por tanto, mediante el resultado se puede identificar que no tiene una representatividad significativa, sino que se torna mayormente hacia el funcionamiento de los sentimientos y emociones percibidas.

3. No se detectó alteraciones cognitivas, significativas, asimismo, los conceptos evolutivos que aparecieron fueron mencionados por los padres de familia como " el porqué no puedes madurar", "el no pude madurar", "no puedo crecer"; esto hace referencia los diversos duelos que explica Aberástury (1971), razón por la cual, es importante establecer líneas de acción para la intervención para que los padres de los menores puedan realizar alternativas propias dentro del hogar.

4. Con lo expuesto, se permite establecer lineamientos para la prevención del suicidio desde una postura efectiva dentro del fenómeno social en la población de adolescentes, de esta forma para abordarla desde una postura multidisciplinaria es necesario la clasificación de riesgos en los menores como lo son:

a. Identificación de "alto riesgo" de realizar conducta suicida; por lo que se debe proceder a una intervención de tipo individualizada y especializada con profesionales del sector de salud mental integrando farmacoterapia, terapia cognitivo conductual o tratamiento conductual de tipo dialéctico.

b. Identificación de subgrupos en la población que pueden estar en estado de vulnerabilidad para desarrollar el acto suicida, como lo son los centros de instituciones escolares. 
c. La fase de desarrollo de prevención global, que es la dirigida a toda la población o denominado nivel universal (Bustamante y Florenzano, 2013).

5. En sumativa, la investigación brinda elementos para identificar factores que están involucrados en la valoración del porqué se realiza el acto suicida en el menor, por ello, las estrategias de intervención y sobre todo de prevención es la posibilidad de brindar alternativas de solución para que puedan tener una mejor adaptabilidad del menor sin llegar al fracaso como dolor psíquico incontrolable en las circunstancias actuales.

\section{References}

Aberastury, A. y Knobel, M. (1971). La adolescencia normal. Un enfoque psicoanalítico. Paidos. Argentina.

Aguilar, P. y Neri, L. (2018). El análisis del discurso como disciplina metodológica en problemáticas sociales: el suicidio y los mensajes póstumos. Consejo Nacional de Ciencias Sociales. COMECSO:México.

Bustamante, F. y Florenzano R. (2013) Programas de prevención del suicidio adolescente en establecimientos escolares: una revisión de la literatura. Revista Chilena de neuro-psiquiatría. 51 (2).

Chávez, M. (s/f). Reportaje cartas póstumas en el suicidio de adolescentes y niños. Revista Contenido.

Instituto Nacional de Estadística y Geografía e Informática (2020). Estadística de mortalidad. Bases de datos CONAPO. México.

Manual Diagnóstico y Estadístico de los Trastornos Mentales. (2018). Guía de Consulta de los Criterios Diagnósticos. DSM-5. Asociación Estadounidense de Psiquiatría. Editorial Médica Panamericana. USA.

Organización Mundial de la Salud (2019). Suicidio. OMS.

Pacheco, B. (2019). Riesgo de la infancia y la adolescencia. Editorial Colofón. México.
(2020). Depresión en Adolescentes.

(2020, 11, 17) recuperado de facebook.com/UnidadAcademicaPsicologia

Quintanar, F. (2007). Comportamiento suicida. Perfil psicológico y posibilidades de tratamiento. Pax. México.

Romero, L. (2015). Planificación suicida.Cartas póstumas. (2020, 12, 29). Recuperado en https://www.facebook.com/psiqforense/posts/7 49027245197018/

Royo, J. y Martínez, M. (2012) Depresión y sucidio en la infancia y adolescencia. Centro de Salud mental Infanto-Juvenil. Revista Pediatría Integral VVI (9), 755-759.

Secretaría de Salud (2020). Cuadernos de salud pública. México. 